\title{
Construction of Nominal Yield Curve for Nairobi Securities Exchange: An Improvement on Monotone Preserving $r(t) t$ Interpolation Method
}

\author{
Lucy Muthoni, Silas Onyango, Omolo Ongati \\ Institute of Mathematical Sciences (IMS), Strathmore University, Nairobi, Kenya \\ Email: lucymuthoni2003@gmail.com
}

Received 18 September 2015; accepted 16 November 2015; published 19 November 2015

Copyright (C) 2015 by authors and Scientific Research Publishing Inc.

This work is licensed under the Creative Commons Attribution International License (CC BY). http://creativecommons.org/licenses/by/4.0/

(c) () Op Open Access

\begin{abstract}
There is no agreed-upon method used to construct yield curves at the Nairobi Securities Exchange. The existing practice is that each financial company uses in-house methods to construct the yield curves for their pricing and decision making. The most common yield curve used in the market was the one constructed by the Cannon Asset Managers Limited (CAM), a Kenyan company, in 2011. The choice of the interpolation function is extremely important when constructing a yield curve. CAM used linear interpolation on the logarithms of the interest rates as their interpolation function. Studies have shown that all variations of linear interpolations produce discontinuities in the forward rate curve. The monotone convex interpolation method, introduced by Hagan \& West [1], improved on the shortcomings of linear and cubic interpolations by ensuring not only a positive and (mostly) continuous forward rate curve, but also a strictly decreasing curve of discount factors. Unfortunately, the model not only depends heavily on an appropriate interpolation algorithm but also produces discontinuity of $f(t)$ under specific conditions. The monotone preserving $r(t) t$ method improves on monotone convex method in that the knot points are estimated in the manner which ensures positivity and continuity in $f(t)$ besides preserving the geometry of $r(t) t$. Unfortunately, monotone preserving method has the undesirable characteristic of not being differentiable at the knot-points. This paper suggests an improvement on monotone preserving $r(t) t$ interpolation method which ensures that the knot points of the curve are differentiable.
\end{abstract}

\section{Keywords}

Yield Curves, Interpolation Methods, Nairobi Securities Exchange 


\section{Introduction}

\subsection{Background Information}

The Nairobi Securities Exchange (NSE) started trading in shares while Kenya was still a British colony in the 1920s, according to IFC/CBK [2]. Share trading was initially conducted in an informal market that would facilitate access to long-term capital by private enterprises and also allow commencement of floating of local registered Government loans. In 1954, the NSE was constituted as a voluntary association of Securities brokers registered under the Societies Act [3]. The newly established Securities exchange was charged with the responsibility of developing the Securities market and regulating trading activities. Despite its long presence, the securities market is yet to make significant contribution in the country's development process, perhaps due to its slow development path.

The development path of Securities markets in both the emerging and developed world indicates an evolutionary process where changes in institutional infrastructure and the policy environment are witnessed as efforts are made to facilitate the growth of the Securities market. The evolutionary process is also characterized by a shift in trading system from a periodic auction system to a continuous trading system.

Trading systems define the price discovery process or the transformation of latent demand of investors into realized transactions [4]. The evolutionary process of trading systems also indicates a shift from manual and decentralized settlement clearing systems to electronic and centralized settlement clearing. ${ }^{1}$ It is argued that such a trading system enhanced efficiency in the price discovery process, provided liquidity at low costs, and had no excess volatility, thus was more desirable for the development of the Securities market [5] [6]. High liquidity enhances long-term investment by reducing the required rate of returning and lowering the cost of capital to the issuers of securities. An efficient price discovery process enhances the role of the market in aggregating and conveying information through price signals, therefore making prices more informative.

Since the initiation of the Nairobi Securities Exchange in the 1920s, the development of the institutional and policy changes which have affected the market can be summarized as an evolutionary process with three stages of development. The initiation stage was mainly characterized by no formal rules and regulations and was dominated by foreign investors. In the formalization stage, a self-regulatory system was adopted while attempts were made to increase the participation of local investors by the post independent Government. In the third stage, various institutional and policy reforms were implemented to enhance the growth of the market.

Two main factors that shaped the development path were first, the political environment both at the local level and in the East African region which saw change in the policy environment and changed the composition of market participation; and secondly the macroeconomic environment which instigated the demand for locally-mobilized long term capital to enhance economic development.

Considering the developments in various aspects of the market, the following patterns were evident. First, the Securities brokerage industry expanded and diversified with the number of Securities brokers increasing from 6 to 21 (as at September 2014), and licensing of Securities dealers and investment banks also increased. However, Securities brokerage is yet to be fully negotiable while the role of Securities brokers remains predominately that of an agent.

Second, the composition of market participants shows a shift from a market dominated by foreign investors in the initiation stage to increased participation of local investors in the formalization stage (especially in the post-independence period), re-entry of foreign investors, though at limited level and mass education on Securities market operations and assets, in the revitalization stage.

Third, the NSE served as a regional market for the East African states in the initial stages but the Securities Exchange lost a significant proportion of its market scope due to the political changes in these states. Attempts are however underway to establish a regional market to facilitate expansion of the NSE market.

Fourth, though the diversity of securities traded is still minimal, the reform period saw efforts made to attain market depth with introduction of new instruments. Fifth, while a discriminative tax policy that penalized share investors heavily was adopted in the post-independence period, an incentive-based tax policy regime was adopted in the reform period to enhance the competitiveness of the financial assets and reduce the barriers to listing new issues.

Sixth, in an effort to strengthen the regulatory framework, the regulatory system has witnessed a shift from the non-formal system to self-regulatory and statutory regulatory framework. Finally, the trading system shows

${ }^{1}$ Amihud, et al. [5] note the tendency for emerging markets to shift from periodic to continuous trading in the revitalization process. 
a shift from the coffee-house forum to floor trading, and there were attempts to introduce the delivery versus payment system with the introduction of the Central Depository System and automation of the trading cycle. The main aim was to enhance efficiency in the price discovery process and liquidity of the market. These changes mimic development paths of other Securities markets in both the developed and emerging markets.

Many securities markets in emerging markets are on a healthy development path. This has led to a number of Futures Exchanges springing up in Latin America, Asia and Africa, during the last decade, based on the premise that there is a need in the specific countries for a platform to be used to manage price volatility and provide price discovery. However, despite governments' and donor agencies' support, 2 out of 3 contracts traded in these markets fail as they have not been designed properly, according to the Capital Market Authority [7] report. In June 2010 Budget speech, the government of Kenya, through a policy pronouncement by the then Deputy Prime Minister and Minister of Finance, announced that steps would be made towards developing institutional and legal frameworks to introduce a Commodities and Futures exchange in Kenya. This will create a need to design a tool that can be used by the market to price the derivatives properly.

Currently, the NSE does not have a known existing yield curve as indicated by Ngugi et al. [8]. According to Ngugi et al. [8], leading stockbrokers, a group of bank traders and institutional fund managers were working with Reuters Limited in 2006 towards developing an acceptable, credible market yield curve. This was driven by the requirement of International Accounting Standard (IAS) 39, which requires that there should be a standard yield curve to facilitate investors in fixed income instruments to value their portfolios at fair market values. However, the market participants identified limitations in prices reported on the NSE and decided not to use the yield curve created at the time to value their portfolios.

It was then agreed that the yield rates applied to the market were to be derived from a cross-section of key market players. These key market players were required to post their quotes on Reuters' assigned pages, out of which aggregate yields are derived. The yield curve, which was primarily available through Reuters, which is a media channel dedicated to providing market data in the international financial market and disseminating that information locally.

In 2011, Cannon Asset Managers (CAM), a Kenyan based Asset Management company, created a yield curve for the NSE using the rates given by the Central Bank of Kenya (CBK), according to CAM [9]. They used logarithmic linear interpolation method to calculate the yield rates at the periods missing from the CBK's bonds. Unfortunately, logarithmic linear interpolation has a tendency of implying discontinuities in the forward rate curve, a weakness depicted by all variations of linear interpolation methods, as shown by Hagan and West [1].

We intend to construct a yield curve for the NSE by overcoming the limitations experienced by the group working on the yield curve in 2006. The main limitation of their method was using the bonds' dirty prices, as quoted at the NSE. To improve on this, we will use raw bond data from CBK, which is the primary issuer of the bonds in Kenya. This way, we will construct a yield curve which is based on clean bond prices (without accruals of interest and so forth, as is the case in secondary market). In addition, we intend to use an interpolation method which has been verified as being superior to the variations of linear interpolation methods, and that is an improvement of monotone preserving $r(t) t$ method.

\subsection{The Yield Curve}

A yield curve is a graphical representation of relationship between return (yield) of same type of financial instruments and its day to maturity. In other words, all the differences in terms of types, credit risks and liquidity are removed from bonds and just the path of interest rates according to maturity rate is represented in yield curves.

Yield curves can be grouped into two in terms of coupons: namely coupon bearing yield curves and zero coupon yield curve. A coupon bearing yield curve is obtained from an observable market bonds at various times to maturity with the bonds having the same coupon rate. Most of the government securities having long maturity dates usually have coupons. When the bond itself and the coupons traded separately we have what is called STRIPS (Separate Trading of Registered Interest and Principal of Securities), in finance literature. A zero coupon bond yield curve is the yield curve between zero coupon bond yield and maturity dates. Zero coupon yields are the difference between the purchasing price and face value of bonds since the bonds do not pay any coupon until maturity date.

Yield curves can also be grouped into nominal yield curves, spot yields curves, and forward yield curves. 
Nominal yield curves take place in primary bond markets, and it is the graph of yields of bonds which are transacted at nominal prices. Spot yield curve is another definition of zero coupon yield curve. Forward yield curve is the curve representing the connection between the forward rate and its corresponding maturity where the forward rate is the interest rate implied by the zero coupon rates for periods of time in the future.

\subsection{Shapes of Yield Curves}

The yield curves reflect the market expectations and may take one of the three main patterns: normal, flat or inverted curves. Each shape includes different information for market.

\subsubsection{Normal Yield Curve}

As the name indicates, this is the yield curve shape that forms during normal market conditions. Normal market conditions occur when investors generally believe that there will be no significant changes in the economy, such as in inflation rates, and that the economy will continue to grow at a normal rate. During such conditions, investors expect higher yields for fixed income securities with long-term maturities that occur farther into the future.

A normal yield curve is an indication of normal expectation of the market because short-term instruments generally hold less risk than long-term instruments; the farther into the future the bond's maturity, the more time, and therefore, uncertainty, the bondholder faces before being paid back the principal. To invest in one instrument for a longer period of time, an investor needs to be compensated for undertaking the additional risk.

\subsubsection{Flat Yield Curve}

These curves indicate that the market environment is sending mixed signals to investors, who are interpreting interest rate movements in various ways. During such an environment, it is difficult for the market to determine whether interest rates will move significantly in either direction into the future.

A flat yield curve usually occurs when the market is making a transition that emits different but simultaneous indications of what interest rates will do. In other words, there may be some signals that short term interest rates will rise and other signals that long-term interest rates will fall. This condition will create a curve that is flatter than its normal positive trade off by choosing fixed-income securities with the least risk, or highest credit quality.

\subsubsection{Inverted Yield Curve}

These yield curves form when the expectations of investors are completely the inverse of those demonstrated by the normal yield curve. In such abnormal market environments, bonds with maturity dates farther into the future are priced higher than the short-term bonds. This means that the market expects yield of long term bonds to decline.

An inverted yield curve indicates that investors interpret an inverted curve as an indication that the economy will soon experience a slowdown, which causes future interest rates to give even lower yields before a slowdown, thus creating a need to lock money into long-term investments at present prevailing yields, because it is expected that future yield will be even lower.

\subsection{Zero-Coupon Yield Curves}

The zero-coupon yield curve is also known as the term structure of interest rates. It measures the relationship among the yields on default-free securities that differ only in the term to maturity. By offering a complete schedule of interest rates across time, the term structure embodies the market's anticipation of future events.

In bond-valuation, the term structure of interest rates refers to the relationship between bond prices of different maturities in general. When interest rates of bonds are plotted against their maturities, this is called the yield curve. The term structure of interest rates and yield curves are used interchangeably in literature.

\subsection{Theories of the Yield Curves}

\subsubsection{Expectations Theory}

There are various versions of expectations theories. These theories place predominant emphasis on the expected values of future spot rates or holding-period returns. In its simplest form, the expectation hypothesis postulates 
that bonds are priced so that the implied forward rates are equal to the expected spot rates. Generally, this approach is characterized by the following propositions 1) the return on holding a long term bond to maturity is equal to the expected return or repeated investment in a series of the short-term bonds, or 2) the expected rate of return over the next holding period in the same for bonds for all maturities [10].

The key assumption behind this theory is that buyers of bonds do not prefer bonds of one maturity over another, so they will not hold any quantity of a bond if its expected return is less than that of another bond with different maturity. Bonds that have this characteristic are said to be perfect substitutes. Note that what makes long term bonds different from short term bonds are the inflation and interest rate risks. Therefore, this theory essentially assumes away inflation and interest rate risks [11].

Another version of expectations theory holds that the slope of the yield curve reflects only investors' expectations for future short-term interest rates much of the time [12]. Investors expect interest rates to rise in the future, which accounts for upward slope of the yield curve. If the expectations hypothesis were correct, the slope of term structure could be used to forecast the future path of interest rates for example, if the yield curve were to slope upward at the short end, it would be because the interest rate is expected to rise.

One problem with this version of the expectations hypothesis is that in fact, the yield curve slopes upward at the short end on average even though interest rates do not rise on the average. One way to explain divergence is to assume that investors are simply wrong on average. ${ }^{2}$

The expectations hypothesis can easily be modified to account for this persistent upward slope in a way that does not require systematic errors on the part of investors .Since bond prices do fluctuate over time, there is uncertainty(even for default free bonds) regarding the return from holding a long-term bond over the next period. Moreover the amount of uncertainty increases with maturity period of the bond. If there were a risk premium associated with uncertainty, then the yield curve could slope upward on average without implying that interest rates increase on average. If the risk premium were constant, the changes, in the slope of the yield curve would forecast changes in the future path of the interest rate. For example, if the slope of the yield curve were to increase, then it would have to be because the path of futures interest rates is expected to be higher. This increase in the slope would imply that future bond yield would be higher. But there is a problem with this version of the hypothesis as well, according to a number of authors.

Another feature of the yield curve that the expectations has difficulty explaining is that the zero-coupon yield curves slopes downward on average at the long end, typically over the range of twenty to thirty years bond. In other words, the yield on a thirty-year zero-coupon bond is typically below the yield on a twenty-year bond. The expectations hypothesis would suggest that that this slope is due to either 1) a persistently incorrect belief that the interest rate will begin to fall about 20 years from or 2) a decrease in the risk premium for bonds with maturities beyond twenty years, even though the uncertainty of the holding-period return for thirty-year bonds. Neither of this reasons is sensible ${ }^{3}$.

There is, however, a sensible explanation, for the persistent downward slope for the term structure at the long end. The explanation has to do with uncertainty regarding the future-path of short-term rates. This uncertainty underlies the risk of holding bonds (if there were no uncertainty regarding the future paths, there would be no risk of holding default-free bonds.) Increases in this uncertainty lead to 1) increases in risk premia that increase the slope of the yield curve at the short end and 2) decreases in the slope of the yield curve at the long end via the effect of convexity. Convexity (technically known as Jensen's inequality) arises from the non-linear relation between bond yields and bond prices. As a consequence, a symmetric increase in uncertainty about yield raises the average price of bonds, thereby lowering their current yields. This effect is trivial at the short end of the yield curve where it plays no significant role, because it becomes noticeable and even dominant at the long end. The overall shape of the yield curve involves the trade-off between the competing effects of risk premia (which cause longer term yields to be lower). Typically, the maximum yield occurs in the fifteen to twenty-five maturity range of the zero coupon yield. ${ }^{4}$

\footnotetext{
${ }^{2}$ Another way to explain the divergence is to assume that investors give some weight to very large increases in the interest rate that have not yet been observed. This is sometimes called a "peso-problem". But a good theory should not imply that investors are wrong on average.

${ }^{3}$ There is another explanation (not related to the expectations hypothesis) that is sensible. The downward slope at the long end of the yield curve could, in principle, reflect a substantial demand for the longest-maturity (default free) zero coupon bond (for example, to insulate the value of insurance companies' long term liabilities from interest-rate risk).

${ }^{4}$ It should be stressed that the yield curve typically reported in the newspaper is not the zero-coupon yield curve and may display a somewhat different shape owing to a variety of factors according to Fisher [12].
} 


\subsubsection{Segments Market Theory}

This is the market segmentation hypothesis of Culbertson. Here it is asserted that individuals have strong maturity preferences, and that bonds of different maturities trade in separate markets. This theory assumes that markets for different-maturity bonds are completely segmented. The interest rate for each bond with a different maturity is then determined by the supply of and demand for the bond with no effects from the expected returns on other bonds and other maturities. In other words, longer maturity bonds that have associated with the inflation and interest rate risks are completely different assets than the shorter bonds. Thus, the bonds of different maturities are not substitutes at all, so the expected returns from a bond of one maturity has no effect on the demand for a bond of another maturity. Because bonds of shorter holding periods have lower inflation and interest rate risks, segmented market theory predicts that yield on longer bonds will generally be higher, which explains why yield curve is usually upward sloping.

However, since markets for different-maturities bonds are completely segmented, there is no reason why the short and long yield should move together. And, because of the same reason, the segment market theory also cannot explain why the short-term yield should be more volatile than longer-term yields [11].

\subsubsection{The Liquidity Preference Theory}

This theory is an offshoot of the pure expectations theory and it asserts that long term interest rates not only reflect investor's assumptions about future interest rates but also include a premium for holding long term bonds, called the term premium or the liquidity premium. This premium compensates investors for the added risk of having their money tied up for a longer period, including the greater price uncertainty. Because of the term premium, long term bond yields tend to be higher than short-term yields and the yield curve slope upward [13].

\subsection{The Uses of Yield Curves}

The relationship between yield and maturity has critical importance for policy makers, investors and economists. Yield curve can be used for a range of purposes. For example:

- Yield curves are used in forecasting interest rates for different products when their risk parameters are known.

- Yield curves are used mostly by investors to see the differences in yields of different maturities, and to detect if there is arbitrage opportunity.

- By yield curve information, investors can have opportunity of making immunization of their investment portfolios against financial risks if they have to make investment on some determined time of maturity.

- Private sector firms look at yields of different maturities and then choose their borrowing strategy according to information gotten from the yield curve [14].

- The differences in yields for long maturity and short maturities are an important indicator for central bank to use in monetary policy process as shown by Akinci [15]. These differences may show the tightness of the government monetary policy. The differences can be monitored to predict recession coming in next years.

- Yield curves are also used to calibrate no-arbitrage term structure models like the models of Hull [16]; and Ingersoll [10] which are used in pricing different financial products [17].

\subsection{Yield Curves and Bond Prices}

When it comes to zero-coupon bonds, spot rates can be derived directly from observed prices. For coupon-bearing bonds usually their "yield to maturity" or "par yield" only is quoted. The yield to maturity is its internal rate of return, that is the constant interest rate $r_{k}$, that sets its present value equal to its price, and the equation is expressed as:

$$
P_{k}=\sum_{i}^{n} \frac{C F_{i}}{\left(1+r_{k}\right)^{t_{i}}}
$$

where $P_{k}$ is the price of bond $k$ which generates $n$ cash-flows (CFs) at periods $t_{i}(i=1,2, \cdots, n), \quad r_{k}$ are the spot rates applicable on this bond and $t_{i}$ depicts the maturity dates.

These cash flows consist of the coupon payments and the final repayment of the principal or face value. Yields to maturity on coupon bonds of the same maturity but with different coupon payments are not identical. 
Nevertheless, if the cash flow structure of a bond trading at the market (“at par") is known, it is possible to derive from estimated spot rates, the coupon bond's theoretical yield to maturity, i.e. the rate the bond would require in order to trade at its face value ("at par"). Drawing on the spot rates $s_{t, m}$, the price equation can be expressed as:

$$
P_{k}=\frac{C}{\left(1+r_{t_{1}}\right)}+\frac{C}{\left(1+r_{t_{2}}\right)^{2}}+\cdots+\frac{C}{\left(1+r_{t_{n}}\right)^{n}}+\frac{V}{\left(1+r_{t_{n}}\right)^{n}}=\sum_{i=1}^{i=n} \frac{C}{\left(1+r_{t_{i}}\right)^{i}}+\frac{V}{\left(1+r_{t_{n}}\right)^{n}}
$$

where $C$ represents the coupon payments, $V$ represents the repayments of the principal, $t_{i} \mathrm{~s}$ and $r_{i} \mathrm{~s}$ as before. The yield to maturity of a coupon-bearing bond is therefore a geometric average of the spot rates which, in general, varies with the term to maturity.

\subsection{Extraction of Yield Curve from Bond Prices}

To derive the yield curve, the discount function is estimated by applying a (constrained) non-linear optimization procedure to data observed on a trade day. More important than the choice of a particular optimization method (e.g. maximum likelihood, non-linear least squares, generalized method of moments) is the decision whether the (sum of squared) yield or price errors should be minimized. If one is primarily interested in minimizing the yield rates errors, it is suggested to minimize the deviation between the estimated and observed yields. In this case the estimation proceeds in two stages: first, the discount function $d_{t, m}$ is used to compute estimated prices and, secondly, estimated yields to maturity are calculated by solving the following equation for each coupon-bearing bond $k$ [18]

$$
P_{k}=\sum_{i=1}^{m} C \exp \left(-r_{k} i\right)+V \exp \left(-r_{k} m\right)
$$

where $r_{k}$ is the risk free rate.

\section{Models Used for Extraction of Yield Curve}

In practice, only a finite set of income securities trade, very few of which are zero-coupon bonds [19]. Practioners are therefore forced to use yield curves from coupon-paying bonds to extract zero-coupon yield curve. Therefore a nominal yield curve must exist from which the ZCYC can be extracted. This paper concentrates on construction of the nominal yield curve, with its novelty being in the improvement of the interpolation method used to the constructing the curve.

\section{Literature Review}

Interpolation is a method of constructing new data points within the range of a discrete set of known data points (called knot points). The simplest method for interpolating between two points is by connecting them through a straight line. Some variations of linear interpolation are capable of ensuring a strictly decreasing curve of discount factors. However, all the variations of linear interpolation imply discontinuities in the forward rate curve.

In order to produce continuous forward rates curves, researchers introduced cubic methods of interpolation. An example of cubic interpolation algorithm is the cubic Hermite spline. Under cubic Hermite splines, the derivative of the data of each knot point is assumed to be known, and the interpolation function is required to be differentiable. Often, these derivatives will not be known, and will have to be estimated. One method for estimating these derivatives, described by de Boor [20] as the Bessel method involves estimating the derivative though the use of a three point difference formula.

Unfortunately, all the traditional cubic methods are incapable of ensuring strictly positive forward rates, which are synonymous with non-decreasing discount factors, as shown by Hagan and West [1]. Furthermore, some cubic methods have an inherent lack of locality in the sense that a local perturbation of curve input data will cause ringing and cause changes in the data far away from the perturbed data point as shown by Andersen [19].

All variations of linear interpolations were seen to produce discontinuities in the forward rate curve, whilst all variations of cubic interpolations were seen to be incapable of ensuring strictly decreasing discount factors. Non-decreasing discount factors imply arbitrage opportunities, whilst discontinuous forward rates unacceptable from an economic perspective (unless the discontinuities occur on or around meetings of monetary authorities). 
To counter this, a monotone convex interpolation method was developed, which it is claimed to be capable of ensuring a positive and (mostly) continuous forward rate curve [1]. This method proposed by Hagan and West [1], was specifically designed to interpolate yield curve data, and involves fitting a set of quadratic polynomials to a discrete set of estimated instantaneous forward rates. The method is designed such that $f(t)$ preserves the shape of the set of discrete forward rates. The monotone convex method was also seen to be capable of ensuring a strictly decreasing curve of discount factors. Unfortunately, the model depends heavily on an appropriate interpolation algorithm. In addition, it was discovered by du Preez [21] that there were specific conditions under which the interpolation function of the monotone convex interpolation would produce discontinuity of $f(t)$.

This led to the monotone preserving $r(t) t$ method of interpolation, introduced by Preez [21]. Essentially, this method involves applying cubic Hermite interpolation to the $r(t) t$ at the knot points i.e. the values of $f(t)$ at the knot points, are estimated in manner which ensures positivity in $f(t)$. Constructing an interpolating algorithm capable of preserving the monotonicity of the discount factors, was thus sufficient for ensuring positive forward rates.

Monotone preserving $r(t) t$ method is capable of ensuring a positive and continuous forward rate curve, and was designed to preserve the geometry of $r(t) t$. Monotonicity in the discount factors implies monotonicity in the $r(t) t$ which is achieved by applying the work done in the field of shape preserving cubic interpolation, by authors such as Akima [22]; Carlson [23], Hyman [24] and de Boor\& Schwartz [25]. Apart from being an improvement of monotone convex method where it ensured positive forward rates, the monotone preserving $r(t) t$ method was also capable of ensuring continuity of $f(t)$.

In the study by du Preez [21], they found that the monotone preserving $r(t) t$ method to perform slightly better in terms of stability, and continuity of $f(t)$, compared to monotone convex method. This suggests that when bootstrapping, the monotone preserving $r(t)$ method could be the ideal method of interpolation. Unfortunately, monotone preserving method had the undesirable characteristic of not being differentiable at the knot-points.

This paper seeks to introduce a new method of interpolation, which will be an improvement of monotone preserving $r(t) t$ interpolation method suggested by du Preez [21]. This will be done by removing the nondifferentiability at the knot points in the aforementioned method, which is created by use of Hyman monotonicity constraint, which enforces monotonicity. Further, we will check whether the new interpolation method produces a yield curve that produces decreasing discount rate, which is one of the key attributes of a good yield curve.

\section{Methodology}

\subsection{Bond Pricing Formula}

A yield curve is calibrated from a set of instruments trading in the market. The prices of these instruments are related to a discrete set of points along the yield curve. Before calibration, we need to understand how the prices of these instruments are related to these points along the curve.

\section{Elementary Relations}

Let $F(t)$ represent the value that one unit of currency invested at time $t_{0}$, would be worth at time $t$. From elementary calculus it follows that:

$$
F(t)=\lim _{n \rightarrow \infty}\left(1+\frac{r_{t}}{n}\right)^{n t}=\mathrm{e}^{-r_{t} t}
$$

Let $Z(t)$ represents the value at time $t_{0}$ of one unit of currency to be received at time $t$. $Z(t)$ is thus the inverse of $F(t)$ and is referred to as the price at time $t_{0}$, of the zero-coupon bond maturing at time $t$. It follows from Equation (4) that:

$$
Z(t)=\mathrm{e}^{-r_{t} t} .
$$

Assume that an investor can invest $Z\left(t_{1}\right)$ today, in a zero-coupon that pays one unit currency at time $t_{1}$. Furthermore, assume an investor can invest $Z\left(t_{2}\right)$ today, in a zero-coupon bond that pays one unit of currency at time $t_{2}$. From the law of one price it must follow that: 


$$
Z\left(t_{1}\right) * Z\left(t_{0} ; t_{1}, t_{2}\right)=Z\left(t_{2}\right)
$$

where $Z\left(t_{0} ; t_{1}, t_{2}\right)$ represent the price at time $t_{0}$, of a zero-coupon bond to be purchased at time $t_{1}$, for maturity at $t_{2}$. The discount factor $Z\left(t_{0} ; t_{1}, t_{2}\right)$ is called the forward discount factor from $t_{1}$ to $t_{2}$. If $f\left(t_{1}, t_{2}\right)$, represents the continuously compounded rate of interest, as observed at $t_{0}$, that an investor can earn from $t_{1}$ to $t_{2}$ then Equation (5) implies that:

$$
Z\left(t_{0} ; t_{1}, t_{2}\right)=\mathrm{e}^{-f\left(t_{1}, t_{2}\right)\left(t_{2}-t_{1}\right)}
$$

Equation (6) implies that:

$$
Z\left(t_{0} ; t_{1}, t_{2}\right)=\frac{Z\left(t_{2}\right)}{Z\left(t_{1}\right)} .
$$

From Equation (7) and Equation (8), it follows that:

$$
f\left(t_{1}, t_{2}\right)=\frac{r_{\mathrm{t}_{2}} t_{2}-r_{\mathrm{t}_{1}} t_{1}}{t_{2}-t_{1}}
$$

The forward rate $f\left(t_{1}, t_{2}\right)$ is called the discrete forward rate observed at time $t_{0}$ applicable to the period from $t_{1}$ to $t_{2}$. Consider rewriting $t_{1}$ and $t_{2}$ in Equation (9), as $t$ and $t+\epsilon$ respectively. We then define $f(t)$, taking the limit as $\epsilon \rightarrow 0$, and obtain that:

$$
f(t)=\frac{\mathrm{d}}{\mathrm{d} t} r_{t} t
$$

or equivalently:

$$
r_{t}=\frac{1}{t} \int_{0}^{1} f(\tau) \mathrm{d} \tau
$$

The forward rate $f(t)$ is called the instantaneous forward rate observed at time $t_{0}$, applicable to time $t$. Finally, note that if $t \in\left[t_{i-1}, t_{i}\right)$ then it follows from Equation (11) that:

$$
r_{t} t=\int_{0}^{t_{i-1}} f(\tau) \mathrm{d} \tau+\int_{t_{i-1}}^{t} f(\tau) \mathrm{d} \tau=r_{t_{i-1}} t_{i-1}+\int_{t_{i-1}}^{t} f(\tau) \mathrm{d} \tau
$$

\subsection{Interpolation Methods}

\subsubsection{Monotone Convex Interpolation}

Hagan and West [1] introduced the monotone convex method of interpolation. This was one of the first methods specifically designed to interpolate yield curve data. The merits of this method specifically designed for interpolating yield curve data is justified in light of the fact that none of the so-called "traditional" methods are capable of ensuring a forward curve that is both positive and continuous.

\section{1) Suitable Forward Rates}

The monotone convex method is defined on $f(t)$. As such, an initial set of points $f_{i}, i=1,2, \cdots, n$, is required before interpolation can commence. This immediately poses a concern as one would almost never start off with a set of points on the instantaneous forward rate curve. These rates must thus be estimated. Consider the set of discrete forward rates $f_{i}^{d}$ for $i=1,2, \cdots, n$, calculated as:

$$
f_{i}^{d}=\frac{r_{i} t_{i}-r_{i-1} t_{i-1}}{t_{i}-t_{i-1}}
$$

The assumptions underlying $f_{1}^{d}$ is that $t_{0}=r_{0}=0$. Hagan \& West [1] then model $f_{i}$ for $i=1,2, \cdots, n-1$ as the slope at time $t_{i}$, of the quadratic that passes through $\left(t_{i-j}, f_{i-j}^{d}\right)$, for $j=1,0$ and -1 . The instantaneous forward rates at the end points, i.e. $f_{0}$ and $f_{n}$ are chosen so as to ensure

$$
f_{0}^{\prime}=0=f_{n}^{\prime} \text {. }
$$

The instantaneous forward rates are thus estimated as: 


$$
f_{i}=\frac{t_{i}-t_{i+1}}{t_{i+1}-t_{i-1}} f_{i+1}^{d} \frac{t_{i+1}-t_{i}}{t_{i+1}-t_{i-1}} f_{i}^{d}
$$

For $i=1,2, \cdots, n-1$, whilst:

$$
\begin{aligned}
& f_{0}=f_{1}^{d}-\frac{1}{2}\left(f_{1}-f_{1}^{d}\right) \\
& f_{n}=f_{n}^{d}-\frac{1}{2}\left(f_{n-1}-f_{n}^{d}\right) .
\end{aligned}
$$

\section{2) The Basic Interpolator}

Since the monotone convex method is defined as an interpolation method on $f(t)$, we would expect the method to return the input set of instantaneous forward rates $f_{i}$, for $i=0,1, \cdots, n$. Furthermore, we require continuity in $f(t)$, which can be obtained by requiring that $f\left(t_{i}\right)=\left(t_{i}\right)^{2}=f_{i}$, for $i=1,2, \cdots, n-1$, i.e. by requiring that $f\left(t_{i}\right)=f_{i}$ and $f\left(t_{i-1}\right)=f_{i-1}$, for $i=1,2, \cdots, n$. The interpolation function is also required to return the input set of zero-coupon spot rates. Equation (12) implies that:

$$
\begin{aligned}
& r_{t_{i}} t_{i}=r_{t_{i-1}} t_{i-1}+\int_{t_{i-1}}^{t} f(\tau) \mathrm{d} \tau \\
& r_{t_{i}} t_{i}-r_{t_{i-1}} t_{i-1}=\int_{t_{i-1}}^{t} f(\tau) \mathrm{d} \tau . \\
& f_{i}^{d}=\frac{1}{t_{i}-t_{i+1}} \int_{t_{i+1}}^{t} f(\tau) \mathrm{d} \tau
\end{aligned}
$$

It follows that if Equation (18) is satisfied, the method will reproduce the input set of zero-coupon spot rates. The monotone convex method is thus constrained to satisfy:

$$
\begin{gathered}
f\left(t_{i-1}\right)=f_{i-1} \\
f\left(t_{i}\right)=f_{i} \\
f_{i}^{d}=\frac{1}{t_{i}}-t_{i-1} \int_{t_{i-1}}^{t} f(\tau) \mathrm{d} \tau
\end{gathered}
$$

for $i=1,2, \cdots, n$.

Hagan and West [1] then define $x(t)=\frac{t-t_{i-1}}{t_{i}-t_{i-1}}$ for $t_{i-1} \leq t \leq t_{i}$ and postulate:

$$
f(t)=K+L_{x}(t)+M_{x}(t)^{2} .
$$
tions

Equation (22) can be used in conjunction with conditions (18), (19) and (20) to produce the following equa-

$$
\begin{gathered}
f_{i-1}=K \\
f_{i}=K+L+M \\
f_{i}^{d}=\frac{1}{t_{i}-t_{i-1}} \int_{t_{i-1}}^{t_{i}}\left[K+L x(t)+M x(s)^{2}\right] \mathrm{d} s .
\end{gathered}
$$

Note that Equation (25) can be reformulated as

$$
\frac{1}{2} L+\frac{1}{3} M=f_{i}^{d}-f_{i-1} .
$$

Whilst Equation (23), and Equation (24) imply that

$$
L=f_{i}-f_{i-1}-M .
$$

The system of equations implied by Equation (26) and Equation (27) are then easily solved to obtain 


$$
M=3 f_{i}+3 f_{i-1}-6 f_{i}^{d}
$$

and

$$
L=-2 f_{i}-4 f_{i-1}+6 f_{i}^{d} .
$$

The instantaneous forward rate $f(t)$, can thus be written as:

$$
f(t)=\left(1-4 x(t)+3 x(t)^{2}\right) f_{i-1}+\left(-2 x(t)+3 x(t)^{2}\right) f_{i}+\left(6 x(t)+6 x(t)^{2}\right) f_{i}^{d}
$$

for $t_{i-1} \leq t \leq t_{i}$.

\section{3) Enforcing Monotonicity on $f(t)$}

In order to enforce monotonicity of $f(t)$, Hagan and West [1] define

$$
g(x)=f(t)-f_{i}^{d},
$$

from Equation (30) and Equation (31) it follows that:

$$
g(x)=g(0)\left(1-4 x+3 x^{2}\right)+g(i)\left(-2 x^{2}+3 x^{2}\right)
$$

from where:

$$
g^{\prime}(x)=g(0)(1-4+6 x)+g(1)(-2+6 x) .
$$

\section{4) Enforcing Positivity on $f(t)$}

Equation (33) implies that $f$ will be positive if

$$
g(x) \geq f_{i}^{d}
$$

assume that $f \geq 0$, for $i=1,2, \cdots, n$, and that $f_{i} \geq 0$, for $i=0,1, \cdots, n$, i.e. assume the input set of discount factors are strictly decreasing.

\section{5) Continuity in $f(t)$}

This section illustrates a potential weakness of the monotone convex method; after altering the interpolation function to preserve the Monotonicity of $f(t)$, the method no longer guarantees continuity in $f(t)$. Consider the scenario where $g(0)=0$. The interpolation function is given by:

$$
g(x)= \begin{cases}A+(g(0)-A) \frac{(\eta-x)^{2}}{\eta} & 0<x<\eta \\ A x=\eta & \\ A+(g(1)-A)\left(\frac{x-\eta}{1-\eta}\right)^{2} & \eta<x<1\end{cases}
$$

where:

$$
\begin{gathered}
n=\frac{g(1)}{g(1)+g(0)} \\
A=-\frac{g(1) g(1)}{g(0)+g(1)} .
\end{gathered}
$$

Under this particular scenario where $\eta=1$ and $A=0$, it means that $g(x)=0$, for $x \in(0,1)$; this implies that $f(t)=f_{i}^{d}$ for $t_{i-1} \leq t \leq t_{i}$. The problem that arises is, unless $f_{i}^{d}=f_{i}, f(t)$ will have a discontinuity at $t_{i}$, resulting in:

$$
f\left(t_{i}\right)^{-}=f_{i}^{d} \neq f_{i}=f\left(t_{i}\right)^{+}
$$

\subsubsection{Monotone Preserving $r(t) t$ Interpolation}

Much research was done in the 1970s and 1980s in the field of shape preserving cubic Hermite interpolation. Akima [22], Carlson [22] and de Boor [25] are but a few authors that attempted to construct a cubic interpolation function that is capable of preserving the monotonicity of its inputs. du Preez [21] applied the theory of 
monotone preserving cubic interpolation in order to develop an arbitrage free method for interpolating yield curve data. They labeled the method the monotone preserving $r(t) t$ method.

\section{1) The Interpolation Function}

Consider an interpolation function of the following form:

$$
r(t) t=a_{i}+b_{i}\left(t_{i-1}\right)+c_{i}\left(t_{i-1}\right)^{2}+d_{i}\left(t_{i-1}\right)^{3}
$$

where $t_{i} \leq t \leq t_{i+1}$.

The parameters $a_{i}, b_{i}, c_{i}$ and $d_{i}$ are to be estimated in a way that ensures that the interpolation function preserves the monotonicity of $r(t) t$. This was done by finding the interpolation function that satisfies the following conditions:

- All input rates must be reproduced. The interpolation function must thus satisfy: $r\left(t_{i}\right)=r_{i} t_{i}$ for $i=1,2, \cdots, n$;

- The interpolation function must satisfy: $r_{i} t_{i}^{+}=r_{i} t_{i}^{-}$for $i=1,2, \cdots, n$, to ensure that the continuity of spot rates;

- We require continuity in the forward rate curve, i.e. the interpolation function must satisfy $f_{i}^{+}=f_{i}^{-}$, for $i=1,2, \cdots, n$;

- We require that the interpolated yield curve be arbitrage free, i.e. $r(t) t$ must be monotone increasing.

\section{2) The Monotonicity Region for $r(t) t$}

A simple generalization of what was recognized by de Boor [25], namely that if the data is locally increasing at $t_{i}$, and if $f_{i} \leq 3 \min \left(f_{i+1}^{d}, f_{i}^{d}\right)$, then $r(t) t$ will be monotone in the interval $\left(t_{i}, t_{i+1}\right)$, for $i=1,2, \cdots, n-1$, [24]. Carlson [23] independently developed the same Monotonicity condition. du Preez [21] used the analysis developed by Carlson [23] to prove the Monotonicity region for $r(t) t$. In du Preez [21], $f(t)$ is given by:

$$
f(t)=b_{i}+2 c_{i}\left(t-t_{i}\right)+3 d_{i}\left(t-t_{i}\right)^{2}
$$

Where the coefficients are calculated as:

$$
b_{i}=f_{i} ; c_{i}=\frac{-2 f_{i},-f_{i+1}+3 f_{i+1}^{d}}{h_{i}} ; d_{i}=\frac{f_{i},+f_{i+1}-2 f_{i+1}^{d}}{h_{i}^{2}},
$$

using numerical methods. So, we have:

$$
f(t)=f_{i}+\frac{2\left(-2 f_{i},-f_{i+1}+3 f_{i+1}^{d}\right)}{h_{i}}\left(t-t_{i}\right)+\frac{3\left(f_{i},+f_{i+1}-2 f_{i+1}^{d}\right)}{h_{i}^{2}}\left(t-t_{i}\right)^{2}
$$

for $t_{i} \leq t \leq t_{i+1}$, whilst $f^{\prime}(t)$ is given by:

$$
f^{\prime}(t)_{i}=2 c_{i}+6 d_{i}\left(t-t_{i}\right)=\frac{2\left(-2 f_{i},-f_{i+1}+3 f_{i+1}^{d}\right)}{h_{i}}+\frac{6\left(f_{i},+f_{i+1}-2 f_{i+1}^{d}\right)}{h_{i}^{2}}\left(t-t_{i}\right) .
$$

In order to establish the Monotonicity condition implied by interpolation function, Du Preez [21] distinguished between three different scenarios:

$$
\begin{aligned}
& \text { 1. } f_{i}+f_{i+1}-2 f_{i+1}^{d}=0 \\
& \text { 2. } f_{i}+f_{i+1}-2 f_{i+1}^{d}<0 . \\
& \text { 3. } f_{i}+f_{i+1}-2 f_{i+1}^{d}>0
\end{aligned}
$$

The only scenario that requires further analysis is where $f_{i}+f_{i+1}-2 f_{i+1}^{d}>0$, the third scenario that met their conditions. Under this particular scenario $f(t)$ has a local minimum at:

$$
\begin{aligned}
& t^{*}=t_{i}+\frac{h_{i}\left(2 f_{i}+f_{i+1}-3 f_{i+1}^{d}\right)}{3 f_{i}+f_{i+1}-2 f_{i+1}^{d}} \\
& f\left(t^{*}\right)=f_{i}-\frac{2 f_{i}+f_{i+1}-3 f_{i+1}^{d}}{3 f_{i+1}+f_{i}-2 f_{i+1}^{d}}
\end{aligned}
$$


$r(t) t$ will be monotone increasing in $\left(t_{i}, t_{i+1}\right)$ if the following conditions is satisfied: for $t^{*}<t_{i}$ or $\left.t^{*}>t_{i+1}\right)$,

$$
f\left(t^{*}\right) \geq 0 \text {. }
$$

\section{3) Extrapolation}

For extrapolation to be possible, it is required that $f$ be constant before $t_{1}$ and after $t_{n}$. More specifically, that $f(t)=f_{i}$ when $t<t_{1}$, and $f(t)=f_{n}$ when $t>t_{n}$. From Equation (12) it follows that:

$$
r(t) t=r_{0} t_{0}+\int_{0}^{t} f_{1} \mathrm{~d} t=f_{1} t
$$

when $t<t_{1}$.

Similarly, it is required that:

$$
r(t) t=r_{n} t_{n}+\int_{t_{n}}^{t} f_{n} \mathrm{~d} t=r_{n} t_{n}+f_{n}\left(t-t_{n}\right)
$$

when $t>t_{n}$.

\subsubsection{Improvement of Monotone Convex Interpolation on $r(t) t$}

We start with a mesh of data points $\left\{t_{1}, t_{2}, \cdots, t_{n}\right\}$ (we will think of the $\mathrm{x}$-values as time points on the $\mathrm{x}$ axis) and the corresponding $y$ values are define as $\left\{f_{1}, f_{2}, \cdots, f_{n}\right\}$ for a generic but unknown function $f(t)$. Cubic splines are generally defined by piece-wise cubic polynomial that passes through consecutive points:

$$
f(t)=a_{i}+b_{i}\left(t-t_{i}\right)+c_{i}\left(t-t_{i}\right)^{2}+d_{i}+\left(t-t_{i}\right)^{3} .
$$

With $t \in\left[t_{i}, t_{i+1}\right]$ and $i=1, \cdots, n$. We will use the following definitions

$$
\begin{gathered}
h_{i}=t_{i+1}-t_{i} \\
m_{i}=\frac{f_{i+1}-f_{i}}{h_{i}} .
\end{gathered}
$$

With $i=1, \cdots,(n-1)$ The coefficients $a_{i}, b_{i}, c_{i}$ and $d_{i}$ depends on the details of the method, and are related to the values of $f(t)$ and its derivatives at the node points. In general

$$
a_{i}=f\left(t_{i}\right) \equiv f_{i}, b_{i}=f^{\prime}\left(t_{i}\right) \text { and so on }
$$

where in the above equation, the prime denotes the derivative of the interpolating function $f(t)$ w.r.t. its argumentt. Moreover given $a_{i}$ and $b_{i}$, we can express $c_{i}$ and $d_{i}$ as follows:

$$
\begin{gathered}
c_{i}=\frac{3 m_{i}-b_{i+1}-2 b_{i}}{h_{i}} \\
d_{i}=\frac{b_{i+1}-b_{i}-2 m_{i}}{h_{i}^{2}} .
\end{gathered}
$$

We can use (47) to compute the derivative

$$
\frac{\partial f(t)}{\partial f_{j}}=\frac{\partial a_{i}}{\partial f_{j}}=\frac{\partial b_{i}}{\partial f_{j}}\left(t-t_{i}\right)+\frac{\partial c_{i}}{\partial f_{j}}\left(t-t_{i}\right)^{2}+\frac{\partial a_{i}}{\partial f_{j}}\left(t-t_{i}\right)^{3} .
$$

We have:

$$
\begin{aligned}
& \frac{\partial a_{i}}{\partial f_{j}}=\delta_{i}^{j} \\
& \frac{\partial m_{i}}{\partial f_{j}}=\frac{1}{h_{i}} \delta_{i+1}^{j}-\frac{1}{h_{i}} \delta_{i}^{j} \\
& \frac{\partial c_{i}}{\partial f_{j}}=\frac{1}{h_{i}}\left(3 \frac{\partial m_{i}}{\partial f_{j}}-\frac{\partial b_{i+1}}{\partial f_{j}}-2 \frac{\partial m_{i}}{\partial f_{j}}\right)
\end{aligned}
$$




$$
\frac{\partial d_{i}}{\partial f_{j}}=\frac{1}{h_{i}^{2}}\left(\frac{\partial b_{b+1}}{\partial f_{j}}-\frac{\partial b_{i}}{\partial f_{j}}-2 \frac{\partial m_{i}}{\partial f_{j}}\right)
$$

which depends on the matrix element $\frac{\partial b_{i}}{\partial f_{j}}$.

Here $\delta_{i}^{j}$ is the Kronecker delta, which is equal to one if $i=j$ and zero otherwise. Once the derivatives at the points, or equivalently the $b_{i}$ coefficients, are specified, everything else is fixed. In particular we are interested in computing $\frac{\partial f(t)}{\partial f_{j}}$, then all the work will be in the calculation of the derivatives $b_{i}$ w.r.t $f_{j}$.

This calculation is tricky if we use monotone preserving splines (or any other method which enforces monotonicity where the $b_{i}$ are non-differentiable functions of the $f_{j}$ 's (which involve the min and max functions). And this is where the novelty of this paper comes in.

Let us start by recalling the formulas for the $b_{i}$ 's in the monotone preserving cubic spline method as defined in the Hagan-West [1]. First of all, at the boundaries, we have:

$$
b_{i}=0, b_{n}=0 .
$$

For the internal data, if the curve is not a monotone at $t_{i}$, i.e. $m_{i-1} \cdot m_{i} \leq 0$, then the boundaries become:

$$
b_{i}=0\left(\text { if } m_{i-1} \cdot m_{i} \leq 0\right) .
$$

So that it will have a turning point there. Instead if the trend is a monotone at $t_{i}$, i.e. $m_{i-1} \cdot m_{i}>0$, then one defines:

$$
\beta_{i}=\frac{3 m_{i-1} \cdot m_{i}}{\max \left(m_{i-1}, m_{i}\right)+2 \min \left(m_{i-1}, m_{i}\right)}
$$

And:

$$
b_{i}=\left\{\begin{array}{l}
\min \left(\max \left(0, \beta_{i}\right), 3 \min \left(m_{i-1}, m_{i}\right)\right) \text { if } m_{i-1} \cdot m_{i}>0 \\
\min \left(\max \left(0, \beta_{i}\right), 3 \min \left(m_{i-1}, m_{i}\right)\right) \text { if } m_{i-1} \cdot m_{i}<0
\end{array} .\right.
$$

The former choice is made when the curve is increasing (positive slopes), the latter when decreasing (negative slopes). Equation (61) represents the monotonicity constraint introduced by Hyman and based on the FritschButland algorithm.

We can now move to the monotonicity constrain. What we need first is:

$$
\begin{gathered}
\frac{\partial}{\partial f_{j}} \max \left(0, \beta_{i}\right)= \begin{cases}0 & \text { if } \beta_{i}<0 \Leftrightarrow m_{i-1}, m_{i}<0 \\
\frac{\partial \beta_{i}}{\partial f_{j}} & \text { if } \beta_{i}>0 \Leftrightarrow m_{i-1}, m_{i}>0\end{cases} \\
\frac{\partial}{\partial f_{j}} \min \left(0, \beta_{i}\right)= \begin{cases}0 & \text { if } \beta_{i}>0 \Leftrightarrow m_{i-1}, m_{i}>0 \\
\frac{\partial \beta_{i}}{\partial f_{j}} & \text { if } \beta_{i}<0 \Leftrightarrow m_{i-1}, m_{i}<0\end{cases}
\end{gathered}
$$

The final step consists of putting all the information together to compute $\frac{\partial b_{i}}{\partial f_{j}}$. Suppose first that the trend is increasing, i.e. $m_{i-1}, m_{i}, \beta_{i}>0$. Using (3.58) we find:

$$
\frac{\partial b_{i}}{\partial f_{j}}=\frac{\partial}{\partial f_{j}}\left[\min \left(\max \left(0, \beta_{i}\right), 3 * \min \left(m_{i-1}, m_{i}\right)\right)\right]
$$




$$
=\left\{\begin{array}{ll}
\frac{\partial}{\partial f_{j}} \max \left(0, \beta_{i}\right) & \text { if } \max \left(0, \beta_{i}\right)<3 * \min \left(m_{i-1}, m_{i}\right) \\
3 * \frac{\partial}{\partial f_{j}} \min \left(m_{i-1}, m_{i}\right) & \text { if } \max \left(0, \beta_{i}\right)>3 * \min \left(m_{i-1}, m_{i}\right)
\end{array} .\right.
$$

Let us now suppose that the trend is decreasing instead, i.e. $m_{i-1}, m_{i}, \beta_{i}<0$. By equation (61) we have:

$$
\begin{aligned}
& \frac{\partial b_{i}}{\partial f_{j}}=\frac{\partial}{\partial f_{j}}\left[\max \left(\min \left(0, \beta_{i}\right), 3 * \max \left(m_{i-1}, m_{i}\right)\right)\right] \\
& =\left\{\begin{array}{ll}
\frac{\partial}{\partial f_{j}} \min \left(0, \beta_{i}\right) & \text { if } \min \left(0, \beta_{i}\right)>3 * \max \left(m_{i-1}, m_{i}\right) \\
3 \frac{\partial}{\partial f_{j}} \max \left(m_{i-1}, m_{i}\right) & \text { if } \min \left(0, \beta_{i}\right)<3 * \max \left(m_{i-1}, m_{i}\right)
\end{array} .\right.
\end{aligned}
$$

It can be shown that for any $i$ and $j$

$$
\begin{gathered}
\frac{\partial}{\partial f_{j}} \max \left(m_{i-1}, m_{i}\right)= \begin{cases}\frac{\partial m_{i-1}}{\partial f_{j}} & \text { if } m_{i-1}>m_{i} \\
\frac{\partial m_{i}}{\partial f_{j}} & \text { if } m_{i-1}<m_{i}\end{cases} \\
\frac{\partial}{\partial f_{j}} \min \left(m_{i-1}, m_{i}\right)= \begin{cases}\frac{\partial m_{i-1}}{\partial f_{j}} & \text { if } m_{i-1}<m_{i} \\
\frac{\partial m_{i}}{\partial f_{j}} & \text { if } m_{i-1}>m_{i}\end{cases}
\end{gathered}
$$

This formula solves our problem of non-differentiability found in the monotone preserving convex on $r(t) t$

\section{Conclusion}

The monotone preserving $r(t) t$ method improves on monotone convex method in that the knot points are estimated in the manner which ensures positivity and continuity in $f(t)$ besides preserving the geometry of $r(t) t$. Unfortunately, monotone preserving method has the undesirable characteristic of not being differentiable at the knot-points. This paper has been able to derive an improvement on monotone preserving $r(t) t$ interpolation method which ensures that the knot points of the curve are differentiable, by removing the non-differentiability found at the max/min points as shown below:

$$
\frac{\partial b_{i}}{\partial f_{j}}= \begin{cases}\frac{\partial}{\partial f_{j}} \min \left(0, \beta_{i}\right) & \text { if } \min \left(0, \beta_{i}\right)>3 * \max \left(m_{i-1}, m_{i}\right) \\ 3 \frac{\partial}{\partial f_{j}} \max \left(m_{i-1}, m_{i}\right) & \text { if } \min \left(0, \beta_{i}\right)<3 * \max \left(m_{i-1}, m_{i}\right)\end{cases}
$$

where: $\frac{\partial}{\partial f_{j}} \max \left(m_{i-1}, m_{i}\right)=\left\{\begin{array}{ll}\frac{\partial m_{i-1}}{\partial f_{j}} & \text { if } m_{i-1}>m_{i} \\ \frac{\partial m_{i}}{\partial f_{j}} & \text { if } m_{i-1}<m_{i}\end{array}\right.$ and $\quad \frac{\partial}{\partial f_{j}} \min \left(m_{i-1}, m_{i}\right)=\left\{\begin{array}{ll}\frac{\partial m_{i-1}}{\partial f_{j}} & \text { if } m_{i-1}<m_{i} \\ \frac{\partial m_{i}}{\partial f_{j}} & \text { if } m_{i-1}>m_{i}\end{array}\right.$.

This formula solves our problem of non-differentiability found in the monotone preserving convex on $r(t) t$, which was the main objective of this paper. This result is important for practical as well as conceptual reasons. Moreover this quantity is important in many areas (e.g. in finance for pricing and for risk management of interest rate derivatives). 


\section{References}

[1] Hagan, P.W.G. (2006) Methods for Constructing a Yield Curve. Wilmott Magazine, 70-81.

[2] IFC/CBK (1984) Development of Money and Capital Markets in Kenya.

[3] NSE (1997) Annual Report 1997. Nairobi.

[4] Madhavan, A. (1992) Trading Mechanism in Securities Market. Journal of Finance, 607-641. http://dx.doi.org/10.1111/j.1540-6261.1992.tb04403.x

[5] Amihuda, Y., Mendelsonb, H. and Lauterbachc, B. (1997) Market Microstructure and Securities Values: Evidence from Tel Aviv Stock Exchange. Journal of Financial Economics, 45, 365-390. http://dx.doi.org/10.1016/S0304-405X(97)00021-4

[6] Bessembinder, H. and Kaufman, H.M. (1997) A Cross Exchange Comparison of Execution Costs and Information Flow for NYSE Listed Stock. Journal of Financial Economics, 46, 293-320. http://dx.doi.org/10.1016/S0304-405X(97)00032-9

[7] Capital Market Authority (2013) Policy Framework and International Best Practices for Establishment and Operations of a Futures/Derivatives Market.

[8] Ngugi, et al. (2007) Development of Bond Market: Kenya’s Experience. KIPPRA Working Paper No. 15.

[9] Cannon Asset Managers (2011) Kenyan Treasury Yield Curve and Bond Valuation.

[10] Cox, J.C., Ingersoll Jr., J.E. and Ross, S.A. (1985) A Theory of the Term Structure of Interest Rates. Econometrica, 53, 385-408.

[11] Munasib, et al. (2003) Money, Banking and Financial Markets. 8th Edition, Econ 3313, 1-8.

[12] Fisher, M. (2001) Forces That Shape the Yield Curve. Federal Reserve Bank of Atlanta, Economic Review First Quarter, 2-5.

[13] Management, Pacific Investment (MPI) (2004) PIMCO, LLC, 840 Newport Center Drive, Newport Beach, CA 92660: PIMCO, 1-8.

[14] Teker, et al. (2004) Hazine Bonosuve Eurobonds Uzerine Uygulama. Ulusal Finans Sempozyumu, 8.

[15] Akinci, O.G. (2006) Devlet ic Borclanma Senetleri icin Getiri Egrisi Tahmini. TCMB Arastirma ve Para Politikasi Genel Mudurlugu. Calisma Tebligi No: 06/08.

[16] Hull, W. (1990) Pricing Interest Rate Derivative Security. The Review of Financial Studies, 3, 573-592. http://dx.doi.org/10.1093/rfs/3.4.573

[17] Place, J. (2000) Basic Bond Analysis Handbooks in Central Bank.

[18] Svensson, L. (1992) Estimating and Interpreting Forward Interest Rates. NBER Working Paper Series 4871.

[19] Anderson, L. (2007) Discount Curve Construction with Tension Spline. Notes on Review of Derivatives Research, 10, 227-267.

[20] de Boor, C. (2001) A Practical Guide to Splines (Applied Mathematical Sciences). Springer-Verlag.

[21] du Preez, P.F. (2011) An Investigation into Popular Methods for Constructing Yield Curves.

[22] Akima, H. (1994) A New Method of Interpolation and Smooth Curve Fitting Based on Local Procedures. Journal of the Association for Computing Machinery, 52-62.

[23] Carlson, F. (1980) Monotone Piecewise Cubic Interpolation. SIAM Journal of Numerical Analysis.

[24] Hyman, J. (1990) Accurate Monotonicy Preserving Cubic Interpolation. SIAM Journal of Scientific and Statistical Computing, 4, 645-654. http://dx.doi.org/10.1137/0904045

[25] de Boor, C.S.B. (1977) Piecewise Monotone Interpolation. Journal of Approximation Theory, 21, 411-416. 